DOI: https://doi.org/10.11144/Javeriana.upsy18-2.jppr

\title{
Juego patológico en población reclusa colombiana: resultados preliminares en prevalencia y comorbilidad*
}

\section{Pathological Gambling in Colombian Offenders: Early Results on Prevalence and Comorbidity}

Recepción: 16 Abril 2016 | Aceptación: 22 Enero 2019

\author{
José Ignacio Ruiz-Pérez a \\ Universidad Nacional de Colombia, Colombia \\ ORCID: http://orcid.org/0000-0002-8996-9736 \\ ENRIQUe ECHEBURÚA-OdrIOZOLA \\ Universidad del País Vasco, España \\ ORCID: http://orcid.org/0000-0001-7654-0781
}

\begin{abstract}
a Autor de correspondencia. Correo electrónico: jiruizp@unal.edu.co
\end{abstract}

Para citar este artículo: Ruiz-Pérez, J. I., \& EcheburúaOdriozola, E. (2019). Juego patológico en población reclusa colombiana: resultados preliminares en prevalencia y comorbilidad. Universitas Psychologica, 18(2), 1-15, https://doi.org/10.11144/Javeriana. upsy18-2.jppr

\section{RESUMEN}

El objetivo de este trabajo fue conocer la prevalencia de probable ludopatía en una muestra no aleatoria de reclusos hombres y mujeres $(N=334)$, localizados en cinco centros penitenciarios. Se empleó un cuestionario en dos versiones. La parte común a ambas formas incluyó el Cuestionario Breve de Juego Patológico ([CBJP]; FernándezMontalvo, Báez, \& Echeburúa-Odriozola, 1995), una Lista de Chequeo de Consumo de Sustancias Psicoactivas (Ruiz-Pérez, 2015) e información sociodemográfica. La forma A del cuestionario incorporó la Escala de Impulsividad de Plutchik (Ruiz-Pérez, 2015); la forma B, el cuestionario de evaluación de síntomas de psicopatología SA-45 (Sandín, Valiente, Chorot, Santed, \& Lostao, 2008). Los resultados mostraron que la proporción de probables jugadores patológicos fue del $32.3 \%$ para hombres y del $18.8 \%$ para mujeres. El puntaje en el SA-45 y el consumo de alcohol se asociaron directamente con los síntomas de juego patológico. En cuanto al análisis de regresión, en las mujeres, las variables predictoras de la ludopatía fueron la participación en juegos de azar y el consumo de alcohol; en los hombres, fue el grado de psicopatología, sobre todo la hostilidad.

Palabras clave

juego patológico; psicopatología; drogas; impulsividad.

\begin{abstract}
The aim of this research was to know prevalence of pathological gambling in male and female offenders from five jails. Sample was non-randomized $(\mathrm{N}=334)$. Two forms of a survey were applied. The Brief Questionnaire of Pathological Gambling (BQPG, Fernández-Montalvo, Báez \& EcheburúaOdriozola, 1995), a Check List of Drugs Consumption (Ruiz-Pérez, 2015), and questions of sociodemographic backgrpund were common to each forms. Also, Form A included Plutchik' Impulsivity Scale (in Ruiz-Pérez, 2015) and Form B included the pathology Symptoms Assessment (SA-45) questionnaire (Sandín, Valiente, Chorot, Santed \& Lostao, 2008). Results showed that probable pathological gamblers were $32,3 \%$ of men and $18,8 \%$ of women. Global score in SA-45 and alcohol consumption were
\end{abstract}


associated directly with pathological gambling scores. In a regression analysis level, for women inmates, predictive variables of pathological gambling were gambling activities participation and alcohol consumption. For men, psychopatology was the unique predictor variable, with hostility as main dimension.

Keywords

pathological gambling; psychopathology; drugs; impulsivity.

Diferentes autores llaman la atención sobre la participación en juegos de azar de la población reclusa, antes y durante el encierro, por sus implicaciones en la gestión del tiempo libre en prisión (McEvoy \& Spirgen, 2012; Weatherly, Montes, Peters, \& Wilson, 2012) y por la posible relación entre dicha participación y el padecimiento en algunos reclusos/as de una adicción a los juegos de azar (Lesieur, 1994; Turner, Preston, McAvoy, \& Gillam, 2013). De esta manera, es necesario preguntarse cómo la institución penal maneja o gestiona las actividades de juego de apuestas que se dan entre la población reclusa, siendo este un aspecto al que tradicionalmente se le ha prestado poca atención (McEvoy \& Spirgen, 2012).

Una parte de los reclusos/as reproduce de la calle alguna forma de juego de azar (Turner et al., 2013), debido a factores tales como las restricciones de actividades entre la población reclusa, el poco contacto con el exterior o la necesidad de distraerse de estímulos aversivos de la prisión (Weatherly et al., 2012), Estas actividades no suelen ser reguladas por la institución, a pesar de que pueden afectar al bienestar psicosocial de los internos. Por ejemplo, para Williams (2008), los juegos de azar constituyen una forma de ocupar el tiempo libre en prisión, permiten romper con la monotonía en la percepción del tiempo, fomentan emociones positivas y posibilitan el despliegue de ciertas habilidades personales, facilitando un cierto locus de control interno frente al externo, que en general es fomentado por la vida en prisión. Por otro lado, una falta de regulación institucional de estas actividades puede llevar a riesgos para la convivencia en forma de riñas debido a los resultados en el juego, a deudas que se trata de cubrir con robos o saldar con lesiones y, en relación con el tema central de este trabajo, a mantener o incentivar la adicción a los juegos de azar.

La ludopatía implica la presencia de cuatro o más de los siguientes síntomas: pensar constantemente en el juego, necesidad de apostar cantidades de dinero cada vez mayores, fracaso en intentos de dejar de jugar, irritabilidad al tratar de interrumpir el juego, jugar para escapar de otros problemas o de sentimientos negativos, volver a jugar para recuperar lo perdido en apuestas anteriores, mentir a otros sobre el grado de implicación en el juego, pérdida de relaciones afectivas, educativas o laborales por jugar y/o pedir apoyo en el entorno sociolaboral para responder a las deudas de juego (Temcheff, Derevensky, \& Paskus, 2011), descartando episodios de manía (American Psychiatric Association [APA], 2013). Este trastorno es más frecuente en la población reclusa que en la general (Lesieur, 1994; Nixon, Leigh, \& Nowatzki, 2006; May- Chahal, Reith, Wilson, \& Paton, s. f.; Weatherly et al., 2012), lo cual muestra lo relevante de incluirlo como parte de la evaluación e intervención penitenciarias. Concretamente, en Estados Unidos, se han encontrado proporciones de jugadores con probable ludopatía: 5.2 \% (Walters, 1997), 26 \% (Templer, Kaiser, \& Siscoe, 1993), 30 \% (Emshoff, Zorland, Mooss, \& Perkins, 2008), 17.4 a 32.7 \% (Cuadrado \& Lieberman, 2012); en Canadá: entre 7.8 y $13.4 \%$ (Turner et al., 2013) y entre el 6.3 y $13 \%$ (Turner, Preston, Saunders, McAvoy, \& Jain, 2009), según el instrumento empleado; $6.6 \%$ en Alemania (Zurhold, Verthein, \& Kalke, 2014) y en Nueva Zelanda, entre 16 a $21 \%$ (Abbott, McKenna, \& Giles, 2005). En Latinoamérica, se carece de este tipo de estudios, por lo que un primer objetivo de este trabajo ha sido realizar una aproximación inicial a la prevalencia de vida de ludopatía en población reclusa colombiana. En este sentido, para conocer la prevalencia de la ludopatía, en la mayoría de los estudios mencionados se ha empleado el South Oaks Gambling Screen (SOGS) como principal herramienta de evaluación, como instrumento único (p. ej., Abbot et al., 2005; Walters, 1997) o acompañada 
de otras pruebas basadas en el DSM-IV-TR (Turner et al., 2009; Turner et al., 2013). Algunos estudios plantean el empleo de medidas breves de juego patológico, como el Lie/Bet Questionnaire, de dos ítems (Cuadrado \& Lieberman, 2012; Götestam, Johansson, Wenzel, \& Simonsen, 2004; Zurhold et al., 2014). Otras medidas breves de la ludopatía, que podrían emplearse como medidas de filtro (screening), son el Brief Biopsychosocial Gambling Screen (Gebauer, LaBrie, \& Shaffer, 2010) o el Cuestionario Breve de Juego Patológico ([CBJP], en Fernández-Montalvo \& Echeburúa-Odriozola, 1997), derivado del SOGS y diseñado para población hispanoparlante.

De otro lado, la presencia de la adicción a los juegos de azar se ha relacionado con la de desórdenes de personalidad o trastornos afectivos, como manía (Brooker, Clara, \& Cox, 2009), depresión y distimia (Wom Kim, Grant, Eckert, Faris, \& Hartman, 2006), desorden bipolar (Hodgins, Currie, Currie, \& Fick, 2009), trastorno límite y evitador (Nelson \& Oehlert, 2008), trastorno obsesivo-compulsivo (Rodríguez-Guarín \& Gómez-Restrepo, 1998), trastorno antisocial de la personalidad (Cunningham-Williams, Ben Abdallah, Calallan, \& Cotter, 2007; Echeburúa-Odriozola \& Fernández-Montalvo, 2008; Ledgerwood \& Petry, 2010) o con esquizofrenia (EcheburúaOdriozola, Gómez, \& Freixa, 2017). De acuerdo a ello, un segundo objetivo de este trabajo es conocer las relaciones entre síntomas de ludopatía y psicopatología, en población reclusa colombiana. Al respecto, la prueba Symptoms Assessment -45 (SA-45) tiene la ventaja de explorar como filtro la presencia de nueve ejes de psicopatología de forma relativamente breve (Sandín, Valiente, Chorot, Santed, \& Lostao, 2008).

En esta misma línea, se han encontrado evidencias de relaciones entre adicción a juegos de azar y consumo de drogas y alcohol (Abbot et al., 2005; Echeburúa-Odriozola \& Fernández-Montalvo, 2005; Emshoff et al., 2008; Petry, 2005; Shaffer et al., 2007; Wanner, Vitaro, Carbonneau \& Tremblay, 2009; Winters, Stinchfield, Botzet, \& Slutske, 2005). En este sentido, Echeburúa-Odriozola, GonzálezOrtega, Corral y Polo-López (2011) señalan que alrededor del $15 \%$ de las personas con problemas de alcoholismo son también jugadores patológicos, mientras que el $50 \%$ de estos últimos presentan problemas de alcohol. Es así como la asociación entre consumo de alcohol y juego patológico se puede dar por varias vías: antes de jugar: consumir alcohol para evitar la culpa y la autocrítica por jugar; durante el juego, para potenciar la estimulación y después de jugar, para superar el malestar por las pérdidas. Dada la relevancia de este tema, también constituye un tercer objetivo de este trabajo analizar las relaciones entre consumo de drogas y ludopatía en población reclusa. Para ello, empero, hay que tener en cuenta que esta relación puede estar mediada por el género. Por ejemplo, para Echeburúa-Odriozola, González-Ortega, Corral y Polo-López (2011) y González-Ortega, Echeburúa-Odriozola, Corral, Polo-López y Alberich (2013), existe una asociación directa entre juego patológico y depresión y consumo de alcohol solo en mujeres, mientras que en los hombres el juego se asocia con consumo de alcohol y con búsqueda de sensaciones.

Por último, debido a la relación que se ha encontrado entre delitos no violentos o contra la propiedad y la ludopatía en muestras de reclusos (Cuadrado \& Lieberman, 2012; Weatherly et al., 2012) o la consideración de la ludopatía como factor causal del delito (Abbott et al., 2005; Rosenthal \& Lorenz, 1992; Turner et al., 2013; Zurhold et al., 2014), en el actual trabajo, se exploran posibles vinculaciones entre tipos de delitos con niveles altos de síntomas de ludopatía.

\section{Método}

\section{Diseño y muestra}

Se trata de un diseño de investigación descriptivo y correlacional, enfocado a conocer la prevalencia de sintomatología asociada al juego patológico y sus relaciones con variables penitenciarias y de consumo de drogas. La 
muestra fue no aleatoria y estuvo compuesta por 320 personas recluidas en alrededor de cinco establecimientos penitenciarios, escogidos dentro de un recorrido con finalidad académica por las regiones nororiental, central y suroccidental del país, de acuerdo a la clasificación geográfica de establecimientos del Instituto Colombiano Penitenciario y Carcelario (INPEC). Este recorrido consistió en visitar cada establecimiento para realizar la aplicación de los instrumentos seleccionados en el marco de la investigación (véase más adelante, Materiales e instrumentos). Los establecimientos penales y los grupos de reclusos/as cubiertos fueron seleccionados por disponibilidad, voluntariedad y en función de la proximidad a las ciudades que conformaron el recorrido. En la Tabla 1, se presenta la caracterización sociodemográfica y judicial-penitenciaria de la muestra. Predominan en el conjunto de la población los delitos relacionados con micro o macro tráfico de drogas, homicidio y hurto, cubriendo estos tres delitos el $48 \%$ de los internos que proporcionaron información sobre este aspecto.

\section{Tabla 1}

Descripción de la muestra en aspectos sociodemográficos y penitenciarios, a nivel general y por sexos

\begin{tabular}{lrrr}
\hline \multicolumn{1}{c}{ Variable } & \multicolumn{1}{c}{ Total } & \multicolumn{1}{c}{$\begin{array}{c}\text { Hombres } \\
\mathbf{( 5 3 . 2 \% )}\end{array}$} & \multicolumn{1}{c}{$\begin{array}{c}\text { Mujeres } \\
\mathbf{( 4 6 . 8 \% )}\end{array}$} \\
\hline Edad (media y desviación estándar) & $37.6(12.6)$ & $38(12.9)$ & $37.1(\mid 1.8)$ \\
\% Escolaridad hasta primaria & $147(45.2)$ & $68(39.4)$ & $79(52)$ \\
\% Clase baja & $258(72.9)$ & $135(70)$ & $123(76.4)$ \\
\% Solteros & $152(40.4)$ & $77(38.3)$ & $75(42.9)$ \\
$\quad$ Delitos & & & \\
\% homicidio & $49(14.2)$ & $29(16.3)$ & $20(12)$ \\
\% hurto & $46(13.4)$ & $24(13.5)$ & $22(13.3)$ \\
\% porte ilegal armas & $22(6.4)$ & $15(8.4)$ & $7(4.2)$ \\
\% extorsión & $20(5.8)$ & $9(5.1)$ & $11(6.6)$ \\
\% concierto delinquir & $35(10.2)$ & $23(12.9)$ & $12(7.2)$ \\
\% drogas & $104(30.2)$ & $32(18)$ & $72(43.4)$ \\
\% delito sexual & $29(8.4)$ & $19(10.7)$ & $10(6)$ \\
\% secuestro & $10(2.9)$ & $9(5.1)$ & $1(0.6)$ \\
\% otros & $15(8.4)$ & $9(10.1)$ & $6(6.6)$ \\
\hline
\end{tabular}

Las muestras de hombres y mujeres son equivalentes en edad, nivel de escolaridad y socioeconómico y estado civil. En relación con el delito, entre las mujeres es mayor la proporción de condenadas por delitos relacionados con las drogas, mientras que en los hombres son ligeramente mayores las proporciones por homicidio, porte ilegal de armas, concierto para delinquir, principalmente $\left[\chi^{2}(13, \mathrm{~N}: 90)=43.65\right.$

$\mathrm{p}<0.001]$

\section{Materiales e instrumentos}

Se elaboró un cuestionario para recolectar la información de interés que contenía las secciones siguientes de variables e instrumentos, además de la información sociodemográfica mostrada en los párrafos anteriores (edad, sexo, nivel socioeconómico, estado civil, delito de la condena actual).

\section{Sintomatología de juego patológico}

Se midió con el Cuestionario Breve de Juego Patológico ([CBJP]; Fernández-Montalvo, Báez, \& Echeburúa-Odriozola, 1995), que consta de cuatro ítems de formato dicotómico, utilizable como una medida de screening de la ludopatía. Cubre aspectos de autopercepción de problemas con los juegos de azar, culpabilidad experimentada por jugar, tomar dinero de familia o amistades para jugar y dificultad de dejar de jugar. La fiabilidad interna de este instrumento fue de 0.95 en el estudio original, combinando sujetos clínicos y control, con validez discriminante para diferenciar ambos grupos, fiabilidad test-retest y validez convergente con el SOGS; en estudiantes técnicos/tecnológicos y universitarios colombianos, la fiabilidad interna del CBJP fue de 0.73 (Ruiz-Pérez \& Echeburúa-Odriozola, 2017), similar a la del presente trabajo. Los criterios tenidos en cuenta para escoger el CBJP como la escala para medir los síntomas y prevalencia de la ludopatía en el presente estudio fueron su relativa brevedad, siendo para población con, frecuentemente, bajas competencias de lectoescritura, la existencia de estudios previos con población general colombiana con este instrumento, y por tanto, la posibilidad de realizar comparaciones de resultados entre la muestra actual y las de esos estudios anteriores (RuizPérez \& Echeburúa-Odriozola, 2017). 
Lista de Chequeo de Consumo de Sustancias Psicoactivas (Ruiz-Pérez, 2015)

Explora el consumo de cinco sustancias: cigarrillo, alcohol, marihuana, cocaína y bazuco y medicinas y otras sustancias. Para cada sustancia se formulan tres preguntas: 1) si se ha consumido alguna vez en la vida (respuesta dicotómica, SÍ/ NO), 2) la edad del primer consumo y 3) la frecuencia de consumo anterior y durante la estancia en prisión, con cuatro alternativas de respuesta: nunca, una vez al mes, una vez a la semana, casi todos los días. De nuevo, la relativa brevedad de este formato, junto a su uso anterior con poblaciones similares por parte del primer autor, fue el criterio para escogerlo para el actual estudio.

Por otro lado, con el objetivo de no saturar a las personas encuestadas con un instrumento relativamente largo, se confeccionaron dos versiones, que se aplicaron aleatoriamente por establecimiento. En la forma A se incluyó, además de las anteriores secciones, la Escala de Impulsividad de Plutchik (Ruiz-Pérez, 2015). Consta de 15 ítems, con cuatro opciones de respuesta, sobre la secuencia (de nunca a casi siempre) de realización de diversas conductas impulsivas. La escala arroja un puntaje en impulsividad y ha mostrado una aceptable fiabilidad interna (0.74) en una muestra de reclusos (Ruiz-Pérez \& Rodríguez, 2011) y de 0.76 en la actual muestra. En la segunda versión (Forma B), se incluyó una medida de psicopatología, la SA-45 (Sandín et al., 2008). Se presenta como una versión abreviada del SCL-90, empleando cinco ítems para cada una de las dimensiones que mide el instrumento: depresión, hostilidad, sensibilidad interpersonal, somatización, ansiedad, psicoticismo, ansiedad fóbica, obsesión-compulsión e ideación paranoide. En un estudio previo, la fiabilidad interna de cada subescala osciló entre 0.68 y 0.83, excepto para el eje de psicoticismo, con un alfa de Cronbach de 0.55 (Ruiz-Pérez, Garay, \& Muñoz, 2015). La escala ha mostrado validez convergente y divergente con indicadores de psicopatología (Slavin-Mulford, Perkey, Blais,
Stein, \& Sinclair, 2015) y confirmación de una estructura de nueve factores (Alvarado, Sandín, Váldez-Medina, González-Arratia, \& Rivera, 2012). Vale señalar que, del total de la muestra, la forma A fue respondida por el $31 \%$ de la muestra y la forma B por el $69 \%$.

\section{Procedimiento y plan de análisis}

La aplicación de los instrumentos fue autorizada por el INPEC. La participación de los internos/ as fue voluntaria y no se asoció con ningún beneficio penitenciario o de otro tipo. Para ello, se presentó cada instrumento a los internos, explicándoles el objetivo de la investigación, el respeto al anonimato, la voluntariedad en responder la encuesta y recopilando el consentimiento informado. Se aplicó la encuesta en grupos pequeños para ser respondida de forma autoaplicada o mediante entrevista a los sujetos con baja competencia en lectoescritura.

Los datos recogidos de esta manera se digitalizaron y se analizaron con el programa SPSS v. 22 para obtener los índices de fiabilidad interna, calcular los puntajes de cada participante en cada una de las escalas, lo cual permitió establecer, entre otros puntos, los datos de prevalencia vida del juego patológico, comparar las respuestas de hombres y mujeres y conocer las variables explicativas del juego patológico, mediante análisis de correlación y de regresión.

\section{Resultados}

\section{Fiabilidad interna de las escalas}

En cuanto a la fiabilidad interna de las escalas, se encuentra en la mayoría de ellas índices aceptables, particularmente en las dimensiones de salud psicológica (SA-45), de impulsividad y de percepción del mundo. Sin embargo, la dimensión de psicoticismo del SA-45 también presenta un coeficiente muy bajo, por lo que esta dimensión no fue tenida en cuenta en los análisis subsiguientes. En cuanto al Cuestionario 
de Juego Patológico, y de cara a uno de los objetivos de este trabajo, la fiabilidad alcanzada para hombres y mujeres es buena, similar a sujetos colombianos de la población general (RuizPérez \& Echeburúa-Odriozola, 2017), aunque sensiblemente menor de la mostrada en el estudio original (Fernández-Montalvo et al., 1995).

Por otro lado, en las comparaciones entre sexos, se advierte una ausencia de diferencias significativas en la mayoría de las escalas. Las mujeres presentan niveles más altos de psicopatología en general-probablemente por los niveles más altos en somatización y sensibilidad interpersonal- y los hombres mayor presencia de síntomas de juego patológico (Tabla 2).

\section{Tabla 2}

Fiabilidad interna de las escalas y comparación de hombres y mujeres (U de Mann Withney)

\begin{tabular}{|c|c|c|c|c|c|}
\hline Escala & $\alpha(\mathbf{N})$ & Media & Mujeres & Hombres & $\begin{array}{l}\text { U de Mann } \\
\text { Whitney }\end{array}$ \\
\hline Impulsividad (Plutchik) & $0.79(125)$ & 0.87 & 59.01 & 71.64 & $1340+$ \\
\hline SA-45. & $0.95(128)$ & 0.94 & 79.1 & 59.01 & $1116^{* *}$ \\
\hline Depresión & $0.65(185)$ & 1.2 & 96.74 & 90.22 & n. s. \\
\hline Hostilidad & $0.79(186)$ & 0.4 & 95.43 & 92.05 & n. s. \\
\hline Sensibilidad Interpersonal & $0.74(189)$ & 1 & 108.5 & 88.25 & $2781^{*}$ \\
\hline Somatización & $0.86(191)$ & 1 & 114.1 & 87.93 & $2543^{* *}$ \\
\hline Ansiedad & $0.74(183)$ & 1.1 & 103 & 89.29 & n. s. \\
\hline Psicoticismo & $0.57(188)$ & 0.8 & 89.14 & 95.82 & n. s. \\
\hline Obsesión-compulsión & $0.72(193)$ & 1.2 & 100.4 & 94.36 & n. s. \\
\hline Ansiedad föbica & $0.73(189)$ & 0.6 & 100.9 & 91.98 & n. s. \\
\hline Ideación paranoide & $0.64(194)$ & 1.2 & 107.2 & 92.52 & n.s. \\
\hline Juego patológico (KR-20) & $0.73(320)$ & 0 & 149.23 & 180.54 & $11048^{* * * *}$ \\
\hline
\end{tabular}

Prevalencia en juego patológico: análisis por ítems y por clasificación

En relación con el primer objetivo planteado, se calcularon las proporciones de sujetos a) con probable ludopatía, b) con algún riesgo y c) sin problemas en el juego, con base en las puntuaciones en el CBJP (Tabla 3). Hay que tener en cuenta que se emplea una medida de screening aplicada de forma colectiva. También se calcularon las proporciones de quienes respondieron afirmativamente a cada uno de los ítems de la Escala, todo ello para la muestra global y separando por hombres y mujeres (Tabla 3 ).

Se encuentra que algo más del $26 \%$ de la muestra, con un $32 \%$ de hombres y casi el $19 \%$ de las mujeres, podría ser clasificado inicialmente con un riesgo alto de padecer ludopatía. La tasa de prevalencia de síntomas de probable juego patológico es mayor en estas muestras de personas reclusas que en el caso de población colombiana de estudiantes de carreras superiores. La diferencia entre hombres y mujeres es estadísticamente significativa en la clasificación diagnóstica, como lo era en el puntaje total de síntomas reportados. Aunque en todos los ítems del instrumento, los hombres presentan mayor presencia del síntoma, la diferencia entre sexos es significativa solo para el sentimiento de culpabilidad. Vale la pena señalar que, empleando los tres primeros ítems del cuestionario, los porcentajes de sujetos clasificados tentativamente en las categorías diagnósticas son muy parecidos, decreciendo solo un $2 \%$, aproximadamente; los porcentajes de sujetos clasificados como probables jugadores patológicos (sin problemas con los juegos de azar: $57.4 \%$; con algún problema: $18 \%$, probables jugadores patológicos: $24.6 \%$ ), por lo que se podría pensar en emplear la versión de tres ítems en lugar de cuatro (Tabla 4).

Tabla 3

Resultados en el Cuestionario Breve de Juego Patológico: Prevalencias

\begin{tabular}{|c|c|c|c|c|}
\hline Categorías diagnósticas & Total ${ }^{\text {a }}$ & Mujeres & Hombres & $\chi^{2}(1)$ \\
\hline Sin problemas en el juego (0) & $55.3(67.1)$ & $65.3(74.4)$ & $47.6(53.3)$ & \\
\hline Algún problema (1) & $18.3(13.7)$ & $16.0(12)$ & $20.1(16.9)$ & $11.03 * *$ \\
\hline Probable ludopatía (2) & $26.4(19.2)$ & $18.8(13.6)$ & $32.3(29.8)$ & \\
\hline
\end{tabular}

Tabla 4

Resultados en el Cuestionario Breve de Juego

Patológico: Análisis por ítems

\begin{tabular}{lrrrr}
\hline \multicolumn{1}{c}{$\begin{array}{c}\text { Items del CBJP (\% sujetos con } \\
\text { respuesta afirmativa al item) }\end{array}$} & Total & Mujeres & Hombres & $\chi^{2}(\mathbf{1})$ \\
\hline $\begin{array}{l}\text { 1-¿Cree usted que tiene o ha tenido alguna } \\
\text { vez problemas con el juego? }\end{array}$ & 25.6 & 21.9 & 28.5 & 1.95 n. s. \\
$\begin{array}{l}\text { 2-¿Se ha sentido alguna vez culpable por } \\
\text { jugar o por lo que le ocurre cuando juega? }\end{array}$ & 28.7 & 20.1 & 35.2 & $9.39 * *$ \\
$\begin{array}{l}\text { 3-¿Ha intentado alguna vez dejar de jugar } \\
\text { y no ha sido capaz de ello? }\end{array}$ & 27.6 & 23.5 & 30.7 & 2.2 n. s. \\
$\begin{array}{l}\text { 4- ¿Ha tomado alguna vez dinero de casa } \\
\text { para jugar o pagar deudas? }\end{array}$ & 11.8 & 9.3 & 13.8 & 1.7 n. s. \\
\hline \multirow{2}{*}{$* * 0.01$} & & &
\end{tabular}


Validez convergente del CBJP con las medidas de impulsividad y psicopatología

Se calcularon correlaciones de Spearman entre el puntaje en el CBJP con las medidas de impulsividad y en el SA-45, para la muestra total, y por separado para hombres y mujeres (Tabla 5). Para las tres muestras, pero sobre todo en el caso de los hombres, una mayor impulsividad se asoció con mayor número de síntomas de ludopatía. En relación con el indicador global del SA-45, también se halló relación entre más síntomas de psicopatología y de ludopatía. Por subescalas y por sexos, se encontraron patrones diferentes de correlaciones: en los hombres, ocho de las nueve subescalas mostraron relaciones directas con los niveles de síntomas de adicción al juego; en cambio, para el caso de las mujeres, solo la hostilidad y la ideación paranoide se asociaron significativa y directamente con el puntaje en el CBJP.

\section{Tabla 5}

Correlaciones de Spearman entre las variables de impulsividad y psicopatología con el puntaje en el Cuestionario Breve de Juego Patológico

\begin{tabular}{|c|c|c|c|}
\hline \multirow{2}{*}{ Escala } & \multicolumn{3}{|c|}{ CBJP } \\
\hline & Muestra total & Mujeres & Hombres \\
\hline Impulsividad (Plutchik) & $0.31 * * *$ & $0.256^{*}$ & $0.401 *$ \\
\hline SA-45 & $0.261 * * *$ & $0.345^{*}$ & $0.321 * *$ \\
\hline Depresión & 0.12 & $0.285+$ & 0.104 \\
\hline Hostilidad & $0.424 * * *$ & $0.482 * * *$ & $0.459 * * *$ \\
\hline Sensibilidad interpersonal & $0.196 * *$ & 0.172 & $0.283 * * *$ \\
\hline Somatización & 0.118 & -0.119 & $0.287 * * *$ \\
\hline Ansiedad & $0.223 * *$ & $0.288+$ & $0.259 * *$ \\
\hline Obsesión-compulsión & $0.218 * *$ & 0.185 & $0.255^{* *}$ \\
\hline Ansiedad fóbica & $0.205 * *$ & $0.277+$ & $0.225^{*}$ \\
\hline Ideación paranoide & $0.199 * *$ & $0.331 *$ & $0.227 * *$ \\
\hline
\end{tabular}

Consumo de droga, hábitos en juegos de azar y juego patológico

En la Tabla 6, se muestran las estadísticas descriptivas de las muestras del estudio en las variables relacionadas con el consumo de drogas. Por un lado, se halló que la sustancia con mayor número de consumidores se refiere a bebidas alcohólicas, seguidas de cigarrillo, tabaco y marihuana; la sustancia menos consumida es el bazuco. Además, el alcohol es la sustancia que se tiende a consumir más tarde que las otras, para la muestra general, y el bazuco es la de inicio más temprano entre quienes la consumen (la categoría "otras drogas" puede incluir medicamentos legales); en cuanto a la frecuencia de consumo actual, destacan sobre todo las frecuencias más altas del tabaco y de la marihuana.

En cuanto a diferencias de género, no se hallaron diferencias en las proporciones de consumo de drogas, excepto en la categoría de otras drogas $\left[c^{2}(1)=6.3, p<0.05\right]$, en el sentido de que las mujeres serían más consumidoras de otras drogas que los hombres. En cambio, el número total de drogas consumidas es mayor en los hombres $\left[F_{(1,243)}=4.53, p<0.05\right]$. Además, los hombres inician más tempranamente el consumo de sustancias como cigarrillo $\left[F_{(1,243)}=\right.$ $17.45, p<0.001]$, bebidas con alcohol $\left[F_{(1,182)}\right.$ $=4.38, p<0.05]$, marihuana $\left[F_{(1,107)}=6.84, p\right.$ $<0.05]$ y cocaína $\left[F_{(1,72)}=6.42, p<0.05\right]$. Así mismo, la frecuencia de consumo de alcohol es mayor en los hombres $\left[F_{(1,209)}=5.8, p<0.05\right]$.

\section{Tabla 6}

Variables relacionadas con el consumo de sustancias psicoactivas

\begin{tabular}{lrrr}
\hline \multicolumn{1}{c}{ Muestra global } & $\begin{array}{c}\% \\
\text { consumidores }\end{array}$ & $\begin{array}{c}\text { Edad primer } \\
\text { consumo }\end{array}$ & $\begin{array}{r}\text { Frecuencia } \\
\text { de consumo }\end{array}$ \\
\hline -cigarrillo & 52.6 & $13.48(6.5)$ & $2.12(1.52)$ \\
-alcohol & 77.8 & $15.64(5.95)$ & $1.53(1.04)$ \\
-marihuana & 38.1 & $12.38(7.86)$ & $2.06(1.55)$ \\
-cocaína & 24.4 & $10.6(8.8)$ & $1.42(1.27)$ \\
-bazuco & 11 & $9.55(12.7)$ & $1.09(1.31)$ \\
-otras drogas & 7.8 & $4(7.08)$ & $0.76(1.11)$ \\
\hline \multicolumn{1}{c}{ Muestra hombres } & consumidores & Edad primer & Frecuencia \\
consumo & de consumo \\
\hline -cigarrillo & 54 & $11.8(7.1)$ & $2.16(1.56)$ \\
-alcohol & 79 & $14.9(6.31)$ & $1.67(1.07)$ \\
-marihuana & 41.3 & $15.4(6.24)$ & $2.1(1.6)$ \\
-cocaína & 27 & $9.37(8.8)$ & $1.39(1.34)$ \\
-bazuco & 13 & $7.98(12.5)$ & $1.27(0.16)$ \\
-otras drogas & 11.3 & $3.71(7.05)$ & $0.79(1.13)$ \\
\hline Número drogas consumidas & $1.72(1.41)$ & $1.88(1.5)$ & $1.49(1.24)$ \\
(prevalencia vida) & & & \\
\hline \multicolumn{1}{c}{ Muestra mujeres } & consumidores & Edad primer & Frecuencia \\
consumo & de consumo \\
\hline -cigarrillo & 50.7 & $16.1(4.3)$ & $2.07(1.47)$ \\
-alcohol & 75.1 & $18.79(5.15)$ & $1.33(0.96)$ \\
-marihuana & 33.6 & $15.4(6.24)$ & $1.98(1.44)$ \\
-cocaína & 20.5 & $15.6(6.8)$ & $1.47(1.11)$ \\
-bazuco & 8.2 & $16.3(12.04)$ & $1.17(1.43)$ \\
-otras drogas & 2.8 & $6.75(7.89)$ & $0.63(1.09)$ \\
\hline & & &
\end{tabular}

Seguidamente, se exploraron las relaciones entre las variables relacionadas con el consumo de drogas, los hábitos de juegos de azar y las categorías diagnósticas de juego patológico, según los puntajes en el CBJP. De acuerdo a 
ello, el grupo de probables jugadores patológicos se caracteriza en la presente muestra por mayor proporción de consumidores de alcohol (para ambos sexos), de cocaína y bazuco (muestra de hombres), mayor frecuencia de consumo drogas (en los hombres) y mayor proporción de participantes en juegos de azar, aunque con diferencias entre hombres y mujeres en relación con algunas modalidades específicas de juegos (Tabla 7).

A continuación, se llevó a cabo, para cada género, un análisis de regresión paso a paso sobre el puntaje en el CBJP, tomando como variables predictoras aquellas que mostraron un nivel de correlación de $p<0.05$, por cada versión del cuestionario aplicado y por separado para hombres y mujeres. Previamente, se calculó un indicador del total de juegos de azar en los que la persona participó, a partir de la suma de la participación en cartas, lotería, Ganagol, etc.

\section{Tabla 7}

Participación en juegos de azar y uso de drogas según grado de problemas con los juegos de azar

\begin{tabular}{|c|c|c|c|c|}
\hline Mujeres & $\begin{array}{l}\text { Sin riesgo juego } \\
\text { patologico }\end{array}$ & $\begin{array}{l}\text { Algún } \\
\text { riesgo }\end{array}$ & $\begin{array}{c}\text { Probables jugadores } \\
\text { patológicos }\end{array}$ & $\chi^{2}(1)$ \\
\hline \multicolumn{5}{|l|}{ Consumo } \\
\hline Alcohol & 67.6 & 85 & 91.3 & $6.54^{*}$ \\
\hline \multicolumn{5}{|c|}{ Participación en juegos } \\
\hline Cartas & 15.2 & 52.2 & 44.4 & $18.14^{* * *}$ \\
\hline Dados & 4.3 & 26.1 & 37 & $21.7^{* * * *}$ \\
\hline Lotería & 21.7 & 60.9 & 40.7 & $14.28^{* * * *}$ \\
\hline Peleas & 6.5 & 4.3 & 33.3 & $16.32^{* * * *}$ \\
\hline \multicolumn{5}{|l|}{ Animales } \\
\hline Carreras caballos & 1.1 & 4.3 & 14.8 & $9.7 * *$ \\
\hline Otros juegos & 4.3 & 8.7 & $29.6 * * *$ & $15.05^{* * *}$ \\
\hline Hombres & $\begin{array}{l}\text { Sin riesgo juego } \\
\text { patológico }\end{array}$ & $\begin{array}{l}\text { Algún } \\
\text { riesgo }\end{array}$ & $\begin{array}{c}\text { Probables jugadores } \\
\text { patológicos }\end{array}$ & $\chi^{2}(2)$ \\
\hline \multicolumn{5}{|l|}{ Consumo } \\
\hline Alcohol & 73.2 & 88.2 & 92.2 & $8.8^{*}$ \\
\hline Cocaina & 18.3 & 31 & 44.4 & $9.22^{*}$ \\
\hline Bazuco & 4.5 & 11.1 & 29.5 & $14.26^{* * * *}$ \\
\hline \multicolumn{5}{|l|}{ Frecuencia } \\
\hline Cigarrillo & 43.85 & 56.39 & 63.89 & $9.87 * *$ \\
\hline Alcohol & 49.93 & 68.12 & 67.32 & $8.8^{*}$ \\
\hline Cocaina & 28.89 & 37.67 & 43.93 & $7.9^{*}$ \\
\hline Otras drogas & 25.05 & 40.71 & 36 & $10.28^{* *}$ \\
\hline \multicolumn{5}{|c|}{ Participar en juegos } \\
\hline Cartas & 36.6 & 47.2 & 65 & $11.22 * *$ \\
\hline Dados & 23.4 & 42.9 & 51.7 & $12.15^{* *}$ \\
\hline Ganagol & 1.4 & 11.4 & 1.7 & $7.94^{*}$ \\
\hline Otros juegos & 10.5 & 17.6 & 31.7 & $9.66^{* *}$ \\
\hline
\end{tabular}

En los hombres, el puntaje en psicopatología es la variable que predice el nivel de síntomas de juego patológico [Beta estandarizado: 0.585; $\left.t: 3.53, p<0.01 ; \mathrm{F}_{(1,24)}=12.46, p<0.01\right]$, con un porcentaje de varianza explicada del 30 \% (R2 ajustada: 0.314). Cuando se sustituye el puntaje en el SA-45 por el de hostilidad, el porcentaje de varianza explicada es del $27 \%$ (R2 ajustada: 0.268), siendo el puntaje en el CBJP explicado únicamente por la hostilidad (Beta estandarizado: $0.538 ; t: 3.67, p=0.001 ; F_{(1,33)}$ $=13.44, p=0.001]$.

En las mujeres, el nivel de síntomas de ludopatía fue predicho por el total de juegos de azar (Beta estandarizado: 0.582; $t$ : 3.507, $p<$ 0.01) con una varianza explicada del $30 \%$ [R2 ajustado: $\left.0.311 ; F_{(1,24)}=12.3, p<0.01\right] . \mathrm{Al}$ sustituir el puntaje en el SA-45 por la hostilidad, de nuevo es el total de participación en juegos de azar lo que predice el nivel de síntomas en el CBJP (Beta estandarizado: 0.404; $t: 2.61, p<0.05$ ) con una varianza explicada del 14\% [R2 ajustada: $\left.0.139 ; F_{(1,33)}=6.83, p<0.05\right]$. Por otro lado, combinando ambos sexos, y comparando los sujetos sin síntomas de juego patológico y con probable patológico, se obtiene un tamaño del efecto según la d de Cohen de 0.58, por lo que se puede rechazar la hipótesis nula acerca de la ausencia de relaciones significativas entre síntomas de ludopatía y de psicopatología. Al comparar los sujetos con algunos síntomas de ludopatía y aquellos con probable ludopatía, el valor de la d de Cohen es 0.33. Al separar por géneros, comparando sujetos sin problemas con el juego y con probable ludopatía, el valor d es de 0.66 para mujeres y 0.87 para los hombres.

En cuanto a la posible relación entre el delito y nivel de síntomas de ludopatía, en las mujeres no hubo asociación estadísticamente significativa entre ambas variables, siendo los delitos relacionados con drogas (30.8\%), homicidio $(23.1 \%)$ y hurto y delitos sexuales (11.5\% por cada delito) los motivos de condena más frecuentes entre quienes puntuaron como probables jugadores patológicos. En los hombres, la asociación no fue significativa, siendo los delitos de drogas (20.4\%), homicidio (16.7\%), hurto $(16.2 \%)$ y delitos sexuales (13\%) los más frecuentes en los sujetos que puntuaron como probables jugadores patológicos.

\section{Discusión y conclusiones}

El instrumento central para evaluar sintomatología de adicción en el presente estudio, el CBJP, muestra apropiados índices de 
fiabilidad y validez concurrente con la medida de participación en juegos de azar, como lo han mostrado otros estudios (Ladouceur, 2004; RuizPérez, 2014; Toneatto \& Ladouceur, 2003). Así, la fiabilidad interna mostrada por el CBJP es similar a la hallada en estudiantes universitarios (Ruiz-Pérez \& Echeburúa-Odriozola, 2017). El análisis ítem a ítem revela que en todos ellos la prevalencia es mayor entre los hombres que en las mujeres, aunque la diferencia es significativa únicamente para el ítem 2, es decir, el sentimiento de culpa por jugar.

En cuanto al primer objetivo de investigación, de acuerdo al CBJP, alrededor del $26 \%$ de la población reclusa encuestada puntuaría como probable jugadora patológica, con mayor presencia del problema en los hombres que en las mujeres. Este porcentaje es próximo a la estimación de un tercio de la población reclusa que establecen Nixon et al. (2006) en su revisión del tema y a la de Emshoff et al. (2008), aunque superior a los porcentajes hallados en otros trabajos del $5.2 \%$ (Walters, 1997), el $6.6 \%$ (Zurhold et al., 2014) o el 13\% (Turner et al., 2009). Las diferencias de prevalencias probablemente se deban a características de los sujetos y de los centros penales -incluyendo condiciones de encierro, políticas institucionales ante las actividades de ocio de los internos/ as $y / o$ diferencias en la existencia y calidad de programas de tratamiento penitenciario-. En relación con esto, el presente trabajo se basa en la colaboración de sujetos voluntarios, de establecimientos penales de Colombia, de mediana seguridad. De otro lado, la prevalencia vida de probable ludopatía en la muestra del presente estudio es superior a la del 19.2 $\%$ hallada estudiantes colombianos con el mismo instrumento (Ruiz-Pérez \& EcheburúaOdriozola, 2017). Por supuesto, ambas muestras, reclusos/as vs. estudiantes, son cuantitativa y cualitativamente diferentes, aunque por ello llama precisamente la atención que, si bien la muestra del estudio de Ruiz-Pérez y EcheburúaOdriozola (2017) es de edad más joven, con una franja de adolescentes del $21 \%$ (entre los 15 y 18 años), aun en esta submuestra, que es la que tiende a presentar más síntomas de ludopatía que las de edades más altas, la tasa de prevalencia de probables jugadores patológicos $(23.5 \%)$ es inferior a la hallada en el actual estudio. Por otro lado, se suele hallar que la prevalencia de ludopatía es menor en las mujeres respecto a los hombres (Ruiz-Pérez, 2015). Esta diferencia se mantiene en las muestras del presente trabajo, aunque atenuada. Así, se halló casi dos hombres por una mujer con calificación en el CBJP de probable jugador/a patológico. Ello coincide con un estudio de May-Chahal et al. (s. f.), con un $10.4 \%$ de hombres y un $5.9 \%$ de mujeres encarceladas con diagnóstico de jugador problema, frente al $1 \%$ de hombres y $0.1 \%$ de mujeres en la población general.

Acerca del segundo objetivo del trabajo, establecer las relaciones entre sintomatología de ludopatía y de psicopatología, lo que implícitamente se relaciona con la exploración de la validez convergente del CBJP, se halló relaciones significativas entre mayor psicopatología y más síntomas de ludopatía, en consonancia con otros estudios. Por ejemplo, Estévez, Herrero-Fernández, Sarabia y Jáuregui (2015) reportan una relación directa entre puntajes en el SOGS y en el SCL-90R, en adolescentes; Boas de Carvalho, Collakis, Magalhäes y Silveira (2005) encuentran niveles de depresión significativamente más altos en personas adictas a sustancias y a la vez jugadores patológicos que adictos sin problemas en el juego. Ibáñez et al. (2001) hallan en sujetos en tratamiento por ludopatía comorbilidad con algún trastorno psiquiátrico en el $62.3 \%$ de los pacientes, sobre todo desórdenes de personalidad, de adaptación y de abuso o dependencia de sustancias. También se han encontrado niveles altos de psicopatología en pacientes que acuden a una unidad de tratamiento de juego patológico (Jiménez-Murcia et al., 2009). Sin embargo, en el actual trabajo se hallan algunas diferencias llamativas entre hombres y mujeres, ya que del SA-45, en el caso de las mujeres, solo se encuentran correlaciones significativas entre el puntaje en ludopatía con ideación paranoide y con hostilidad, además de con el puntaje total en la escala; en cambio, en los hombres siete de 
ocho subescalas clínicas, además del puntaje total, tienen una correlación directa con el nivel de psicopatología. Estas relaciones se muestran a nivel de correlaciones, de regresiones y en cuanto al tamaño del efecto, siendo entre moderado y alto para los hombres, medio para las mujeres y medio-bajo para la muestra total. Por su lado, el nivel de impulsividad muestra una correlación directa, aunque moderada, con los síntomas de juego patológico. La ausencia de una relación consistente y sólida entre psicopatología y ludopatía en el caso de las mujeres, podría corresponder a la observación de Vachon y Bagby (2009) de que no todos los jugadores patológicos presentan comorbilidad con trastornos psiquiátricos, la que, en todo caso, se asocia con un peor pronóstico del juego patológico (Echeburúa-Odriozola, Amor, \& Gómez, 2017; Parramon, 2015).

Siguiendo en el ámbito de la psicopatología, la hostilidad, en cuanto a correlaciones con los síntomas de adicción al juego, resulta ser la dimensión vinculada con más fuerza, tanto para los hombres como para las mujeres de la muestra del presente estudio. A tenor de su contenido (Slavin-Mulford et al., 2015), esta subescala mide realmente agresión física y verbal e ira, diferente de la hostilidad como desconfianza hacia los demás (Buss \& Perry, 1992). En este sentido, vale la pena recordar que el juego patológico se enmarcaba hasta el DSM-5 (APA, 2013) en el grupo de los trastornos del control de los impulsos, por lo que podría plantearse la existencia de un factor común etiológico del trastorno explosivo intermitente y del juego patológico. En ese sentido, diversos estudios se han centrado en estudiar las relaciones entre conducta violenta y juego patológico (Boughton \& Falenchuk, 2007; Cunningham-Williams et al., 2007). Otra posibilidad es que las tensiones por las deudas debidas al juego, añadidas a la cultura carcelaria de los hombres de mostrarse duros y responder a las ofensas, lleve a algunos reclusos a exhibir una actitud agresiva ante los compañeros de patio. En este sentido, en población general la violencia de pareja puede ser la estrategia de afrontamiento de los jugadores patológicos ante la crisis económica y de pareja que genera la ludopatía (Garrido-Fernández, Jaén Rincón, \& Domínguez Álvarez, 2002).

Por otro lado, con relación al tercer objetivo de explorar las relaciones entre síntomas de ludopatía y consumo de alcohol y otras drogas, los puntajes en el CBJP muestran también relaciones coherentes con varios indicadores de consumo de drogas, aunque de nuevo, con ciertos patrones diferentes por géneros. Las relaciones entre droga, crimen y juego patológico son complejas (Dooley, McCorkle, \& Lukemeyer, 2009), y probablemente no se dan de la misma manera para todos los jugadores de juegos de azar; sin embargo, la literatura es consistente en mostrar comorbilidad entre juego patológico y consumo de sustancias, como el alcohol (Petry, 2005; Shaffer et al., 2007; Slutske, Jackson, \& Sher, 2003; Wanner et al., 2009). En nuestro caso, tanto en mujeres y especialmente en hombres, se halla una correlación significativa entre consumo de sustancias como el alcohol, la cocaína y el bazuco. En los hombres, la prevalencia vida de consumo de estas sustancias y la frecuencia de su consumo anterior al ingreso en prisión se relacionan de forma clara y directa con una mayor sintomatología de juego patológico.

Por último, en relación con el cuarto objetivo, este no se cumple, ya que no se encontró una asociación significativa entre el nivel de síntomas de adicción a juegos de azar con la comisión de algún o algunos delitos específicos. Ahora bien, los datos muestran que las personas reclusas con probable juego patológico están condenadas sobre todo por delitos relacionados con drogas, homicidio, hurto y agresión sexual.

Finalmente, se puede señalar que contar con una medida breve de juego puede ser una estrategia relevante para establecer sondeos de tipo screening, tanto con población normal como en condiciones especiales como lo es la población reclusa. En este sentido, la literatura muestra ejemplos del empleo de medidas de ludopatía aún más breves que la del presente estudio para establecer prevalencias de este trastorno (Cuadrado \& Lieberman, 2012; Zurhold et al., 2014). El CBJP muestra índices satisfactorios de fiabilidad interna y de validez concurrente y convergente, los cuales apuntan a que podría 
constituirse en una prueba de screening útil para el contexto colombiano de prisiones, aunque en el país aún faltan estudios que incluyan comparaciones entre grupos clínicos y de control, tanto para la población en libertad como la encarcelada.

Es así como un diagnóstico individualizado debe apoyarse en un proceso global y riguroso de evaluación; nunca debe sustentarse exclusivamente en los resultados en una prueba de tamizaje. Así, la alta prevalencia hallada en este trabajo en las muestras de mujeres y, especialmente, hombres en prisión, debería ser contrastada con estudios con muestras más amplias y aleatorias, complementados con entrevistas individualizadas. De confirmarse esta alta prevalencia, el juego patológico podría constituirse en uno de los objetivos de intervención de los programas de tratamiento del sistema penitenciario.

\section{Referencias}

Abbott, M. W., McKenna, B. G., \& Giles, L. C. (2005). Gambling and problem gambling among recently sentenced male prisoners in four New Zealand prisons. Journal of Gambling Studies, 21(4), 537-558. https://d oi.org/10.1007/s10899-005-5562-6

Alvarado, B. G., Sandín, B., Váldez-Medina, J. L., González-Arratia N., \& Rivera, S. (2012). Análisis factorial confirmatorio del Cuestionario SA-45 en una muestra mexicana. Anales de Psicología, 28(2), 426-433. https://doi.org/10.6018/analesps. 28.2.148851

American Psychiatric Association. (2013). Diagnostic and statistical manual of mental disorders (5.a ed.). Washington, DC: Autor. https://doi.org/10.1176/appi.books.9 780890425596

Boas de Carvalho, S. V., Collakis, S. T., Magalhäes, M. P., \& Silveira, D. X. (2005). Frequency of pathological gambling among substance abusers under treatment. Revista de Saúde Pública, 39(2), 1-6. https://doi.org /10.1590/S0034-89102005000200012
Boughton R., \& Falenchuk O. (2007). Vulnerability and co-morbidity factors of female problem gambling. Journal of Gambling Studies, 23, 323-334. https://doi.o $\mathrm{rg} / 10.1007 / \mathrm{s} 10899-007-9056-6$

Brooker, I. S., Clara, I. P., \& Cox, B. J. (2009). The Canadian problem gambling index: Factor structure and associations with psychopathology in a nationally representative sample. Canadian Journal of Behavioural Science/Revue Canadienne des Sciences du Comportement, 41(2), 109-114. https://doi.org/10.1037/a0014841

Buss, A. H., \& Perry, M. P. (1992). The Aggression Questionnaire. Journal of Personality and Social Psychology, 63, 452-459. https://doi.org/10.1037/0022-351 4.63.3.452

Cuadrado, M., \& Lieberman, L. (2012). Use of a short gambling screen with an arrestee population: A feasibility study. Journal of Gambling Studies, 28, 193-205. https:// doi.org/10.1007/s10899-011-9253-1

Cunningham-Williams, R. M., Ben Abdallah, A., Callahan, C., \& Cottler, L. (2007). Problem gambling and violence among community-recruited female substance abusers. Psychology and Addict Behavior, 21 (2), 239-243. https://doi.org/10.1037/08 93-164X.21.2.239

Dooley, L., McCorkle, R., \& Lukemeyer, A. (noviembre, 2009). Exploring the temporal ordering of drugs, pathological gambling and criminal offending. Annual Meeting of the ASC Annual Meeting, Filadelfia, Pensilvania.

Echeburúa-Odriozola, E., Amor, P. J., \& Gómez, M. (2017). Current psychological therapeutic approaches for gambling disorder with psychiatric comorbidities: A narrative review. Salud Mental, 40(6), 299-305. https://doi.org/10.17711/sm.0185 $-3325.2017 .038$

Echeburúa-Odriozola, E., \& FernándezMontalvo, J. (2005). Psychological treatment of slot-machine pathological gambling: New perspectives. Journal of 
Gambling Studies, 21(1), 21-26. https://doi. org/10.1007/s10899-004-1918-6

Echeburúa-Odriozola, E., \& FernándezMontalvo, J. (2008). Are there more personality disorders in treatment-seekers pathological gamblers than in other kind of patients? A comparative study between the IPDE and the MCMI. International Journal of Clinical and Health Psychology, 8(1), 53-64. Recuperado de https://www.re dalyc.org/articulo.oa?id $=33780104$

Echeburúa-Odriozola, E., Gómez, M., \& Freixa, M. (2017). Prediction of relapse after cognitive-behavioral treatment of gambling disorder in individuals with chronic schizophrenia: A survival analysis. Behavior Therapy, 48(1), 69-75. https://doi.org/10.10 16/j.beth.2016.09.008

Echeburúa-Odriozola, E., González-Ortega, I., Corral, P., \& Polo-López, R. (2011). Clinical gender differences among adult pathological gamblers seeking treatment. Journal of Gambling Studies, 27(2), 215-227. https://doi.org/10.1007/s10899-0 10-9205-1

Emshoff, J., Zorland, J., Mooss, A., \& Perkins, A. (2008). Gambling and offending: An examination of the literature. Atlanta: Georgia State University.

Estévez, A., Herrero-Fernández, D., Sarabia, I., \& Jáuregui, P. (2015). The impulsivity and sensation-seeking mediators of the psychological consequences of pathological gambling in adolescents. Journal of Gambling Studies, 31(1), 91-103. https://doi .org/10.1007/s10899-013-9419-0

Fernández-Montalvo, J., Báez, C., \& EcheburúaOdriozola, E. (1995). El Cuestionario Breve de Juego Patológico: un nuevo instrumento de "screening". Análisis y Modificación de Conducta, 21(76), 211-223. Recuperado de https://academica-e.unavarra.es/xmlui/ handle/2454/27968

Fernández-Montalvo, J., \& EcheburúaOdriozola, E. (1997). Manual práctico de juego patológico: ayuda para el paciente y guía para el terapeuta. Madrid: Pirámide.
Garrido-Fernández, M., Jaén Rincón, P., \& Domínguez Álvarez, A. M. (2002). Relaciones de pareja y juego patológico: un estudio descriptivo a través de la Escala de Ajuste Diádico (DAS). Apuntes de Psicología, 20(1), 3-17.

Gebauer, L., LaBrie, R. A., Shaffer, H. J. (2010). Optimizing DSM IV classification accuracy: A brief bio-social screen for detecting current gambling disorders among gamblers in the general household population. Canadian Journal of Psychiatry, 55(2), 82-90. https://doi.org/10.1177/0706 74371005500204

González-Ortega, I., Echeburúa-Odriozola, E., Corral, P., Polo-López, R., \& Alberich, S. (2013). Predictors of pathological gambling severity taking gender differences into account. European Addiction Research, 19(3), 146-154. https://doi.org/10.1159/00 0342311

Götestam, K. G., Johansson, A., Wenzel, H. G., \& Simonsen, I. E. (2004). Validation of Lie/ Bet screen for pathological gambling on two normal population data sets. Psychological Reports, 95(3), 1009-1013. https://doi.org/1 0.2466/pr0.95.3.1009-1013

Hodgins, D. C., Currie, S. R., Currie, G., \& Fick, G. H. (2009). Randomized trial of brief motivational treatments for pathological gamblers: More is not necessarily better. Journal of Consulting and Clinical Psychology, 77(5), 950-960. https://doi.org/10.1037/a0 016318

Ibáñez, A., Blanco, C., Donahue, E., Lesieur, H. R., Pérez de Castro, I., Fernández, J., \& SáizRuiz, J. (2001). Psychiatric comorbidity in pathological gamblers seeking treatment. American Journal of Psychiatry, 158(10), 1733-1735. https://doi.org/10.1176/ajp.158 .10 .1733

Jiménez-Murcia, S., Granero Pérez, R., Fernández-Aranda, F., Álvarez Moya, E., Aymamí, M. N., Gómez-Peña, M.,... Mechón, J. M. (2009). Comorbilidad del juego patológico: variables clínicas, personalidad y respuesta al tratamiento. Revista de Psiquiatría y Salud Mental, 2(4), 
178-189. https://doi.org/10.1016/S1888-98 91(09) 73236-7

Ladouceur, R. (2004). Perceptions among pathological and nonpathological gamblers. Adicctive Behaviors, 29, 555-565. https://do i.org/10.1016/j.addbeh.2003.08.025

Ledgerwood, D. M., \& Petry, N. M. (2010). Subtyping pathological gamblers based on impulsivity, depression and anxiety. Psychology and Addictive Behaviors, 24(4), 680-688. https://doi.org/10.1037/a0019906

Lesieur, H. R. (1994). Epidemiological surveys of pathological gambling: Critique and suggestions for modification. Journal of Gambling Studies, 10(4), 385-398. https://d oi.org/10.1007/BF02104904

May-Chahal, C., Reith, G., Wilson, A., \& Paton, K. (s. f). Gambling awareness and problem gambling interventions in UK prisons. Recuperado de http://www.lancaster.ac.uk/ projects/docs/rgsb_strategy_day_cmc.pdf

McEvoy, A., \& Spirgen, N. (2012). Gambling among prison inmates: Patterns and implications. Journal of Gambling Studies, 28(1), 69-76. https://doi.org/10.1007/s1089 9-011-9245-1

Nelson, K.G.; Oehlert, M.E. (2008). Evaluation of a shortered South Oaks Gambling Screen in Veterans with Addictions. Psychology of Addictive Behaviors. 22(2), 309-312. https:/ /doi.org/10.1037/0893-164X.22.2.309

Nixon, G., Leigh, G., \& Nowatzki, N. (2006). Impacting attitudes towards gambling: A prison awareness and a prevention program. Journal of Gambling Studies, 17. ht tps://doi.org/10.4309/jgi.2006.17.14

Parramon, G. (abril, 2015). Gambling disorders and psychotic dual disorders (schizophrenia). En Enrique Echeburúa (Presidencia), Clinical and psychiatric implications of pathological gambling in schizophrenia. Simposio llevado a cabo en el IV International Congress on Dual Disorders, Barcelona.

Petry, N. M. (2005). Stages of change in treatment-seeking pathological gamblers. Journal of Consulting and Clinical Psychology,
73(2), 312-322. https://doi.org/10.1037/a0 035455

Rodríguez-Guarín, M., \& Gómez-Restrepo, C. (1998). Juego patológico: comorbilidad con dependencia de sustancias, trastorno afectivo bipolar y trastorno obsesivocompulsivo. Revista Colombiana de Psiquiatría, 23(3), 181-196. Recuperado de http://psiquiatria.org.co/web/wp-conten t/uploads/2012/04/VOL-27/3/Juego\%20pa tol\%C3\%B3gico.pdf

Rosenthal, R. J., \& Lorenz, V. C. (1992). The pathological gambler as criminal offender: Comments on evaluation and treatment. Psychiatric Clinics of North America, 15(3), 647-660. https://doi.org/10.1016/S0193-95 3X(18)30229-6

Ruiz-Pérez, J. I. (2014). Juego patológico y dependencia del alcohol en una muestra de trabajadores y estudiantes universitarios: prevalencia, interrelaciones y diferencias de género. Psychología: Avances en la Disciplina, 8(1), 33-42. http://www.scielo.org.co/pdf/p sych/v8n1/v8n1a04.pdf

Ruiz-Pérez, J. I. (2015). Adicción a los juegos de azar [Documento interno]. Bogotá: Laboratorio de Psicología Jurídica.

Ruiz-Pérez, J. I., \& Echeburúa-Odriozola, E. (2017). Cuestionario Breve de Juego Patológico en estudiantes colombianos: propiedades psicométricas. Universitas Psychologica, 16(1), 1-12. https://doi.org/10 .11144/Javeriana.upsy16-3.cbjp

Ruiz-Pérez, J. I., Garay, O., \& Muñoz, S. (mayo, 2015). Autoengaño y poligrafía. II Seminario Internacional de Psicofisiología Forense, Bogotá.

Ruiz-Pérez, J. I., \& Rodríguez, L. S. (2011). Análisis psicométrico de una prueba para evaluar actitudes sexistas y hacia la violencia en el ámbito forense: resultados preliminares en una muestra de reclusos. Revista de Psicología Jurídica, 1, 15-20. Recuperado de http://www.humanas.unal.edu.co/psicol ogia juridica/files/3913/6604/8909/Revista PsicoJur.pdf 
Sandín, B., Valiente, R. M., Chorot, P., Santed, M. A., \& Lostao, L. (2008). SA-45: forma abreviada del SCL-90. Psicothema, 20(2), 290-296. Recuperado de http://www.psicot hema.com/pdf/3463.pdf

Shaffer, H. J., Nelson, S. E., LaPlante, D. A., LaBrie, R., Albanese, L., \& Caro, C. (2007). The epidemiology of psychatrics disorders among repeat DVI offenders accepting a treatment sentencing option. Journal of Consulting and Clinical Psychology, 75(5), 795-804. https://doi.org/10.1037/00 22-006X.75.5.795

Slavin-Mulford, J., Perkey, H., Blais, M., Stein, M., \& Sinclair, S. J. (2015). External validity of the Symptom Asessessment Questionnaire (SA-45) in a clinical sample. Comprehensive Psychology, 58, 205-212. https://doi.org/10.1016/j.comppsy ch.2014.12.007

Slutske, W. S., Jackson, K. M., \& Sher, K. J. (2003). The natural history of problem gambling from age 18 to 29. Journal of Abnormal Psychology, 112(2), 263-274. http s://doi.org/10.1037/0021-843X.112.2.263

Temcheff, C. E., Derevensky, J. L., \& Paskus, T. S. (2011). Pathological and disordered gambling: A comparison of DSM-IV and DSM-V criteria. International Gambling Studies, 11(2), 213-220. https://doi.org/10.1 080/14459795.2011.581677

Templer, D. I., Kaiser, G., \& Siscoe, K. (1993). Correlates of pathological gambling propensity in prison inmates. Comprehensive Psychatry, 34(5), 347-351. https://doi.org/1 0.1016/0010-440X(93)90022-V

Toneatto, T., \& Ladouceur, R. (2003). Treatment of pathological gambling: A critical review of the literature. Psychology of Addictive Behaviors, 17(4), 284-292. https://doi.org/1 0.1037/0893-164X.17.4.284

Turner, N. E., Preston, D. L., McAvoy, S., \& Gillam, L. (2013). Problem gambling inside and out: The assessment of community and institutional problem gambling in the Canadian correctional system. Journal of Gambling Studies, 29(3), 435-451. https://d oi.org/10.1007/s10899-012-9321-1
Turner, N. E., Preston, D. L., Saunders, C., McAvoy, S., \& Jain, U. (2009). The relationship of problem gambling to criminal behavior in a sample of Canadian male federal offenders. Journal of Gambling Studies, 25(2), 153-169. https://doi.org/10.1 007/s10899-009-9124-1

Vachon, D. D., \& Bagby, R. M. (2009). Pathological gambling subtypes. Psychological Assessment, 21(4), 608-615. ht tps://doi.org/10.1037/a0016846

Walters, G. D. (1997). Problem gambling in a federal prison population: Results from the South Oaks Gambling Screen. Journal of Gambling Studies, 13(1), 7-24. https://doi.o rg/10.1023/A:1024935115105

Wanner, B., Vitaro, F., Carboneau, R., \& Tremblay, R. E. (2009). Cross-lagged links among gambling, substance use, and delinquency from midadolescence to young adulthood: Additive and moderating effects of common risk factors. Psychology of Addictive Behaviors, 23(1), 91-104. https://d oi.org/10.1037/a0013182

Weatherly, J. N., Montes, K. S., Peters, D., \& Wilson, A. N. (2012). Gambling behind the walls: A behavior-analytic perspective. The Behavior Analyst Today, 13(3-4), 2-8. https: //doi.org/10.1037/h0100725

Williams, D. J. (2008). Offender gambling in prisons and jails: Is it hidden leisure experience?. The Correctional Psychologist, 40, 7-10. https://doi.org/10.11575/PRISM/ 33265

Winters, K. C., Stinchfield, R. D., Botzet, A., \& Slutske, W. S. (2005). Pathways of youth problem severity. Psychology of Addictive Behaviors, 19(1), 104-107. https://doi.org/1 0.1037/0893-164X.19.1.104

Wom Kim, S., Grant, J. E., Eckert, E. D., Faris, P. L., \& Hartman, B. K. (2006). Pathological gambling and mood disorders: Clinical associations and treatment implications. Journal of Affective Disorders, 92, 109-116. h ttps://doi.org/10.1016/j.jad.2005.12.040

Zurhold, H., Verthein, U., \& Kalke, J. (2014). Prevalence of problem gambling among the prison population in Hamburg, 
Juego PATOLÓGICO EN POBLACIÓN RECLUSA COLOMBIANA: RESUlTADOS PRELIMINARES EN PREVALENCIA Y...

Germany. Journal of Gambling Studies, 30 (2), 309-319. https://doi.org/10.1007/s10 899-013-9361-1

\section{Notas}

* Artículo de investigación. 